\title{
In response: Letter on update to the Vitamin C, Thiamine and Steroids in Sepsis (VICTAS) protocol
}

\author{
Christopher J. Lindsell ${ }^{1 *}$, Anna McGlothlin², Samuel Nwosu', Todd W. Rice ${ }^{3}$, Alex Hall ${ }^{4,5}$, Gordon R. Bernard ${ }^{3}$, \\ Laurence W. Busse $^{6}$, E. Wesley Ely ${ }^{7,8,9}$, Alpha A. Fowler ${ }^{10}$, David F. Gaieski ${ }^{11}$, Jeremiah S. Hinson ${ }^{12}$, \\ Michael H. Hooper ${ }^{13}$, James C. Jackson 7,14,15,16, Gabor D. Kelen ${ }^{12}$, Mark Levine ${ }^{17}$, Greg S. Martin ${ }^{5,18}$, \\ Richard E. Rothman ${ }^{12}$, Jonathan E. Sevransky ${ }^{18}$, Kert Viele ${ }^{2}$, David W. Wright ${ }^{4,5}$ and David N. Hager ${ }^{19}$
}

\section{Trial registration}

ClinicalTrials.gov: NCT03509350. Registered on 26 April 2018.

To the Editors,

We thank Drs. Frommelt, Kory, and Long for their interest in the Vitamin C, Thiamine and Steroids in Sepsis (VICTAS) trial and their recommendation to carefully consider time to treatment in our analysis plan. As they have pointed out, our inclusion criteria require study drug administration within $28 \mathrm{~h}$ of the onset of first qualifying organ dysfunction [1]. While it is unknown what differential effect time to hydrocortisone, ascorbic acid, and thiamine (commonly referred to as HAT therapy) may have on patients with sepsis, we agree early treatment with other therapeutics has improved outcomes in sepsis [2]. However, in planning the VICTAS trial, we chose to allow a moderately wide enrollment window reasoning that HAT therapy, if effective, would be of interest to providers managing patients along a wide spectrum of illness severity.

Since completing enrollment in the VICTAS trial in late 2019, discussions of trials exploring the benefits of therapeutic regimens like the one we tested have

This reply refers to the comment available at https://doi.org/10.1186/s13063020-04289-z

* Correspondence: chris.lindsell@vumc.org

${ }^{1}$ Department of Biostatistics, Vanderbilt University Medical Center, Nashville,

TN, USA

Full list of author information is available at the end of the article broadened. While much focus at the time VICTAS was designed was on the question of the efficacy of HAT, negative trials have stimulated a discussion as to when HAT therapy could be effective [3, 4].

In response, we have calculated the time between first qualifying organ dysfunction and first dose of study intervention in the VICTAS trial. This will be reported along with other participant characteristics and will be included in our adjusted analyses following the same approach as described for other covariates. Specifically, we will use restricted cubic splines to address potential nonlinearities, and we will assess for a differential treatment effect. This will be done by testing the interaction between time to treatment and treatment assignment. If the interaction achieves a $P$ value $\leq 0.2$, we will consider the possibility of subgroup analyses with grouping informed by the relationship between time to treatment and outcomes. Since the approach was specified a priori, this helps to mitigate the post hoc addition of this covariate. We expect that including this important variable will strengthen our findings given the suggestion that time to treatment may modify the response to treatment. 


\section{Abbreviations}

HAT: Hydrocortisone, ascorbic acid, and thiamine; VICTAS: Vitamin C, Thiamine and Steroids in Sepsis

\section{Acknowledgments}

None.

\section{Authors' contributions}

All authors provided input to the decisions reflected in this response and approved the letter. All authors read and approved the final manuscript.

\section{Funding}

Funding for VICTAS was provided by the Marcus Foundation via contract to Emory University, the study sponsor. Neither the sponsor nor the funding agency has had any role in the design, execution, or planned analyses for this study. They have had no input on the writing of this letter and will have no input to future updates of the statistical analysis plan. Point-of-care gluc ometers were loaned to some study sites by Nova Biomedical, which has had no role in the design, execution, or planned analyses of the study, nor the writing of this letter.

\section{Availability of data and materials}

A de-identified dataset from participants in the VICTAS trial will be made publicly available approximately 1 year after publication of the primary manuscript.

\section{Ethics approval and consent to participate}

The VICTAS study was approved by a central IRB (Johns Hopkins University IRB protocol number: IRB00164053). Each site's local IRB approved the Informed Consent Document for local use and formally relied on this central approval before enrollment proceeded at that site. This study is registered with ClinicalTrials.gov as NCT03509350.

\section{Competing interests}

CJL reports funding for the VICTAS trial to his institution from the Marcus Foundation, grants, and contracts to his institution from the National Institutes of Health and Endpoint Health and is named as co-inventor on patents related to risk stratification in septic shock. AM and KV are salaried employees of Berry Consultants, which is under contract with Emory University to support the design work and execution of the VICTAS trial. AH reports funding for the VICTAS trial to his institution from the Marcus Foundation as well as grants from the National Institutes of Health $(\mathrm{NIH})$, Cerenovus, Sense Diagnostics, and the NICO Corporation. SN, GRB, DNH, JSH, REM, TR, and RER report funding for the VICTAS trial to their institutions from the Marcus Foundation. EWE reports funding for the VICTAS trial to his institution from the Marcus Foundation and has received honoraria from Pfizer, Orion, and Masimo for continuing medical education activities (no speakers' bureaus or stocks, etc.). ML is supported by the Intramural Research Program, NIDDK, $\mathrm{NIH}$. DK053212-12: Ascorbic acid as a pharmacologic agent in disease treatment. GSM reports grants for the VICTAS Trial to his institution from the Marcus Foundation as well as grants from National Institutes of Health $(\mathrm{NIH})$, Biomedical Advanced Research and Development Authority (BARDA) and Bristol-Myers Squibb to his institution. JES reports grants for the VICTAS trial to his institution from the Marcus Foundation, funding from the Biomedical Advanced Research and Development Authority, and a stipend from the Society of Critical Care Medicine to support his editorial position for the journal Critical Care Medicine. DWW reports the grant for the VICTAS trial to his institution from the Marcus Foundation as well as grants from the National Institutes of Health $(\mathrm{NIH})$, the National Highway Transportation Safety Administration, the Department of Defense, NICO Corporation, and the Centers for Disease Control and Prevention.

\section{Author details}

'Department of Biostatistics, Vanderbilt University Medical Center, Nashville, TN, USA. ${ }^{2}$ Berry Consultants, LLC, Austin, TX, USA. ${ }^{3}$ Division of Pulmonary \& Critical Care Medicine, Department of Medicine, Vanderbilt University School of Medicine, Nashville, TN, USA. ${ }^{4}$ Department of Emergency Medicine, Emory University, Atlanta, GA, USA. ${ }^{5}$ Grady Memorial Hospital, Atlanta, GA, USA. ${ }^{6}$ Division of Pulmonary, Allergy, Critical Care, and Sleep Medicine, Department of Medicine, Emory University, Atlanta, GA, USA. ${ }^{7}$ Division of Pulmonary \& Critical Care, Department of Medicine, Vanderbilt University
Medical Center, Nashville, TN, USA. ${ }^{8}$ Critical Illness, Brain Dysfunction, and Survivorship (CIBS) Center, Vanderbilt University Medical Center, Nashville, TN, USA. 'Tennessee Valley Veteran's Affairs Geriatric Research Education Clinical Center (GRECC), Nashville, TN, USA. ${ }^{10}$ Division of Pulmonary Disease \& Critical Care Medicine, Department of Internal Medicine, The VCU Johnson Center for Critical Care and Pulmonary Research, Virginia Commonwealth University School of Medicine, Richmond, VA, USA. " Department of Emergency Medicine, Sidney Kimmel Medical College, Thomas Jefferson University, Philadelphia, PA, USA. ${ }^{12}$ Department of Emergency Medicine, Johns Hopkins University, Baltimore, MD, USA. ${ }^{13}$ Division of Pulmonary \& Critical Care Medicine, Department of Medicine, Eastern Virginia Medical School and Sentara Healthcare, Norfolk, VA, USA. ${ }^{14}$ Critical Illness, Brain Dysfunction, and Survivorship (CIBS) Center, Vanderbilt University Medical Center, Nashville, TN, USA. ${ }^{15}$ Tennessee Valley Veteran's Affairs Geriatric Research Education Clinical Center (GRECC), Nashville, TN, USA. ${ }^{16}$ Department of Psychiatry, Vanderbilt University School of Medicine, Nashville, TN, USA. ${ }^{17}$ Molecular \& Clinical Nutrition Section, Intramural Research Program, National Institute of Diabetes and Digestive and Kidney Diseases, National Institutes of Health, 10 Center Drive, Bethesda, MD, USA. ${ }^{18}$ Division of Pulmonary, Allergy, Critical Care, and Sleep Medicine, Department of Medicine, Emory University, Emory Critical Care Center, Atlanta, GA, USA. ${ }^{19}$ Division of Pulmonary \& Critical Care Medicine, Department of Medicine, Johns Hopkins University, 1800 Orleans Street, Suite 9121, Baltimore, MD 21287, USA.

Received: 19 March 2020 Accepted: 28 March 2020 Published online: 22 April 2020

\section{References}

1. Hager DN, Hooper MH, Bernard GR, Busse LW, Ely EW, Fowler AA, et al. The Vitamin C, Thiamine and Steroids in Sepsis (VICTAS) Protocol: a prospective, multi-center, double-blind, adaptive sample size, randomized, placebocontrolled, clinical trial. Trials. 2019;20(1):197.

2. Kumar A, Roberts D, Wood KE, Light B, Parrillo JE, Sharma S, et al. Duration of hypotension before initiation of effective antimicrobial therapy is the critical determinant of survival in human septic shock. Crit Care Med. 2006; 34(6):1589-96.

3. Marik PE, Payen D. CITRIS-ALI: How statistics were used to obfuscate the true findings. Anaesth Crit Care Pa. 2019;38(6):575-7.

4. Brant EB, Angus DC. Is high-dose vitamin C beneficial for patients with sepsis? JAMA. 2019;322(13):1257-8.

\section{Publisher's Note}

Springer Nature remains neutral with regard to jurisdictional claims in published maps and institutional affiliations.

Ready to submit your research? Choose BMC and benefit from:

- fast, convenient online submission

- thorough peer review by experienced researchers in your field

- rapid publication on acceptance

- support for research data, including large and complex data types

- gold Open Access which fosters wider collaboration and increased citations

- maximum visibility for your research: over $100 \mathrm{M}$ website views per year

At BMC, research is always in progress.

Learn more biomedcentral.com/submissions 